\title{
AMAZONAS
}

DOI: https://doi.org/10.34069/RA/2021.7.03

Volumen 4, Número 7/enero-junio 2021

\section{El aprendizaje basado en tareas en la expresión oral del idioma inglés al nivel intermedio equivalente a un B1}

\section{Task-based learning in oral skill at the intermediate level equivalent to B1}

Recibido: 10 de enero de $2021 \quad$ Aceptado: 15 de febrero de 2021

\author{
Autores: \\ Georgina Aguilera Saborit ${ }^{10}$ \\ Mirtha Olivero Herrera ${ }^{11}$ \\ Adis Fiol Cuenca ${ }^{12}$
}

\section{Resumen}

La enseñanza-aprendizaje de la expresión oral en el contexto académico supone la implicación del estudiante en situaciones de aprendizaje que exigen el desarrollo de sus potencialidades lingüísticas, determinadas por la existencia, en la práctica, de intereses sociales o profesionales vinculados a la vida real. Sin embargo, aún se observan limitaciones en el desarrollo de esta habilidad comunicativa en la carrera de Ingeniería Geológica, lo que se expresa en su insuficiente autonomía y desempeño interactivo, así como colaborativo. Lo anterior demanda el perfeccionamiento de la didáctica particular del Inglés con Fines Específicos, en la búsqueda de recursos que permitan dinamizar este proceso hacia la independencia cognoscitiva del estudiante. Estos aspectos conducen a la elaboración de las tareas comunicativas presentadas en este trabajo.

Palabras clave: comunicativa, diagnóstico, enseñanza, habilidades.

\begin{abstract}
Teaching-learning of oral skill in academic contexts requires the students' involvement in communicative situations demanding the development of their linguistic potentials, and determined by the existence in practice of social or professional interests related to real life. However, some limitations are still observed in the students' oral communication in Geological Engineering Major expressed on their limited autonomy and oral interactive acting as well as collaborative. The previous idea calls for the improvement of the particular Didactics of English for Specific Purposes, in the search of resources to guide this process towards the students' cognitive independence, originating the preparation of the oral communicative tasks presented in this work.
\end{abstract}

Keywords: communicative, diagnosis, teaching, skill.

\footnotetext{
${ }^{10}$ Licenciada en Lenguas Extranjeras. Vicedecana docente. Profesora Auxiliar. Master en Educación Superior. Departamento de Idiomas, Facultad de Ciencias Económicas, Universidad de Moa; Holguín, Cuba, https://orcid.org/0000-0002-9855-5236

${ }^{11}$ Licenciada en Lenguas Extranjeras. Profesora Auxiliar. Master en Educación Superior. Jefa del Departamento de Idiomas. Facultad de Ciencias Económicas, Universidad de Moa; Holguín, Cuba, https://orcid.org/0000-0002-8221-4410

${ }^{12}$ Licenciada en Lenguas Extranjeras. Profesora Auxiliar. Master en Educación Superior. Departamento de Idiomas, Decana de la Facultad de Ciencias Económicas, Universidad de Moa; Holguín, Cuba, https://orcid.org/0000-0002-0617-7515
} 


\section{AMAZUNAN}

\section{Introducción}

El siglo XXI, época caracterizada por el avance vertiginoso e impacto de la ciencia y la innovación tecnológica, así como el intercambio científico, comercial y cultural, exigen de la universidad cubana el dominio de una lengua extranjera para garantizar la formación de un profesional competente, particularmente el idioma inglés.

En este sentido, el Ministerio de Educación Superior, invoca al perfeccionamiento de la enseñanza del inglés en las universidades cubanas. De ahí, la necesidad de un redimensionamiento del proceso de enseñanza aprendizaje de esta lengua para desarrollar la competencia comunicativa de este idioma.

Este perfeccionamiento exige la adopción del Marco Común Europeo de Referencia para las Lenguas, en lo adelante MCERL, como la base común que orienta la política nacional en lo referente al diseño de estándares, cursos de idioma inglés como lengua extranjera con fines específicos y profesionales (cursos presenciales y/o virtuales), metodologías de enseñanza y materiales didácticos, e instrumentos de evaluación.

Entre los estudiantes que deberán cumplir este requisito de graduación se encuentran, los de la carrera Ingeniería Geológica de la Universidad de Moa, los que deberán demostrar la competencia comunicativa que expresa su capacidad para desarrollar la expresión oral del idioma inglés con eficiencia, al nivel intermedio equivalente a un B1.

Como parte de este proceso en el curso 2019-2020 se aplicaron los exámenes de diagnóstico o colocación y de certificación, donde se constataron las siguientes insuficiencias:

- Reproducen los patrones de ritmo, entonación y acento de la lengua materna.

- En la fluidez del discurso hablado al existir incoherencia en las ideas expresadas y pausas prolongadas.

- En la pronunciación de sonidos vocálicos y consonánticos, por ejemplo el sonido vocálico /I/: se utiliza con la vocal "i" en los vocablos kit, it, big, etc. Este sonido popularmente se denomina como la i (corta). La dificultad que tiene es que no existe en español. En consecuencia, el estudiante no lo reconoce y tiende a pronunciar la i (larga) en las palabras anteriormente señaladas. Eso impacta directamente la habilidad de comprensión al comunicarse.

Los sonidos consonánticos: los estudiantes presentan dificultad al pronunciar palabras que contienen la "th", que puede tener sonidos diferentes como en think o this.

En correspondencia con la problemática existente y para lograr una comunicación eficaz en dicho idioma, de acuerdo con los requerimientos para alcanzar este nivel es necesario la elaboración de tareas comunicativas para desarrollar la expresión oral del idioma inglés, al nivel intermedio equivalente a un B1, en los estudiantes de la carrera Ingeniería Geológica.

\section{Marco Teórico}

\section{La expresión Oral}

La enseñanza y el aprendizaje del idioma inglés en la actualidad se asumen desde un enfoque comunicativo, con un especial énfasis en la expresión oral, considerada la habilidad rectora. 
Numerosos autores han investigado sobre la expresión oral del idioma inglés, entre los que se destacan (Antich, 1986; Abbott, 1989; Byrne, 1989; Brown, 1994; Richards y Lockhart, 1995; Medina, 2006; Font, 2006; Pujol-Breche, 1994 y Acosta, 1996).

$\mathrm{Al}$ respecto Medina (2006) plantea que «la expresión oral es el proceso a través del cual el estudiante-hablante en interacción con una o más personas y de manera activa desempeña un doble papel de receptor del mensaje del (los) interlocutor (es) y de codificador de su mensaje, con el objetivo de satisfacer sus necesidades comunicativas en la lengua extranjera» (Medina, 2006, p.10). El desarrollo de esta habilidad cubre un amplio espectro, desde el enfoque basado en el lenguaje, que enfatiza la exactitud, hasta el basado en el mensaje, que enfatiza el significado y la fluidez. Su objetivo supremo es que el estudiante sea capaz de desarrollar el acto comunicativo con la efectividad requerida. La expresión oral es la habilidad rectora por excelencia en el aprendizaje de lenguas extranjeras. La eficiencia de su desarrollo depende de la integración, con el resto de las habilidades verbales y de un fuerte vínculo entre elementos cognitivos y afectivosmotivacionales, con énfasis en los últimos.

Desde el análisis de estas fuentes, se pudo determinar que sus aportes han estado dirigido a técnicas y procedimientos para el aprendizaje de la expresión oral, el tratamiento a los errores, el papel de la fluidez y la exactitud, la tipología de ejercicios, la necesidad de impartir esta habilidad en un contexto comunicativo, al destacar la competencia comunicativa y sus dimensiones. De modo que, se aprecia una carencia esencial dada en la no instrumentación de tareas comunicativas para el desarrollo de esta actividad verbal, acorde al nivel de asimilación y conocimientos que deben tener los estudiantes para potenciar el enfoque comunicativo.

De modo que, el desarrollo de esta habilidad comunicativa constituye un proceso dual que representa un intercambio dinámico de ideas, experiencias, sentimientos y conocimientos entre emisor y receptor, pequeños grupos y medios de comunicación. Por tanto, se considera la expresión oral como el eje principal de todo el proceso comunicativo, al ser la forma de comunicación más común y usada en el ser humano desde épocas antiguas, la cual refleja diferentes culturas y estilos.

Es válido resaltar, que para el estudiante aprender a expresarse de forma oral debe articular aspectos o niveles esenciales de la lengua como son: la gramática, la fonética y la semántica, todo en función de la fluidez y la exactitud, aspectos necesarios para lograr una competencia comunicativa efectiva.

\section{Aprendizaje basado en tareas}

El Enfoque Basado en Tareas emergió a finales de los años 80 y fue desarrollado por diversos autores como resultado del interés generalizado en la funcionalidad del lenguaje y su enseñanza desde una visión comunicativa.

La enseñanza por tareas constituye lo que Howatt (1984) ha denominado "enfoque comunicativo". La razón es que su objetivo no es sólo enseñar la comunicación como un objeto (como ocurre en el caso del enfoque nocio-funcional) sino lograr que los estudiantes participen en actos auténticos de comunicación en el aula y utilicen el idioma que cursan como una herramienta.

Skehan and Swain (2001) manifiestan que una tarea es «una actividad en la cual los aprendices utilizan el lenguaje para enfatizar en el significado y cumplir un objetivo» (p. 25).

Según Nunan (1989) la tarea en el entorno de aprendizaje de una segunda lengua es «un elemento de trabajo de la clase que implica a los alumnos en comprender, manipular, producir o 


\section{AMAZđ̛́NAS}

interactuar en el lenguaje objeto mientras su atención está enfocada principalmente en el significado más que en la forma» (p.12).

En ese mismo sentido, Ellis (2003) plantea que una tarea «es una actividad que requiere que los alumnos usen el lenguaje poniendo atención no en la forma sino en el significado para conseguir un objetivo.» (p.10)

Por otra parte, señala Willis (2007) que «dentro del marco del aprendizaje basado en tareas, las tareas y los textos se combinan para proporcionar a los alumnos una rica exposición al idioma y también oportunidades para usarlo por sí mismos» (p.33).

Referente a las tareas, por nuestra parte, nos decantamos por las tareas comunicativas. En ese aspecto, Nunan (2011) manifiesta que la tarea comunicativa: “(...) es una parte del trabajo en clase la cual envuelve a los aprendices en la comprensión, manipulación, producción e interacción en la lengua que se está aprendiendo, mientras se centra la atención principalmente en el significado y no en la forma". La tarea debe tener un sentido de complejidad, siendo capaz de permanecer por sí misma como un acto comunicativo.

Las tareas comunicativas son actividades que tienen un objetivo comunicativo, además de constituir una vía material de incorporación de la realidad a la clase donde cobra gran relevancia la interacción, la colaboración, la dinámica del grupo a partir del protagonismo del estudiante, orientado hacia la búsqueda activa del conocimiento, mediante un sistema de actividades que propicien la búsqueda y exploración del conocimiento desde sus posiciones reflexivas, que estimulen el desarrollo de su pensamiento y su independencia cognoscitiva.

\section{Metodología}

Para efectos de este estudio, las autoras de esta investigación proponen el término de tarea comunicativa como unidad de trabajo fundamental en la clase y como medio de interacción sujeto-objeto; o sea entre los estudiantes y el contenido de la lengua, bajo la guía del profesor, donde se centra mayor atención en el significado comunicativo que en la forma en que ocurre el aprendizaje de la lengua inglesa.

En este sentido, se formulan preceptos para elaborar propuestas de tareas comunicativas de carácter general que sirven de base para la dirección del proceso de enseñanza-aprendizaje de la habilidad comunicativa en idioma inglés. En tal sentido la propuesta da respuesta a la necesidad de incrementar la cantidad de estudiantes que cumplen con los requisitos previstos por él, al nivel intermedio equivalente a un B1.

Los preceptos a tener en cuenta para elaborar tareas comunicativas que desarrollen la expresión oral en la enseñanza- aprendizaje del idioma inglés en la carrera Ingeniería Geológica parten de reconocer el papel de los sujetos participantes en el intercambio comunicativo, quienes se convierten en protagonistas de su construcción y transformación., entonces su esencia se expresa en:

\section{Carácter Comunicativo}

Reconoce la constante inserción de los estudiantes en situaciones comunicativas que les permitan ejercitar la formación de los hábitos lingüísticos a través del desarrollo de los diferentes componentes que integran la competencia comunicativa.

Otras de las importantes interrelaciones que se desarrollan en el proceso de enseñanzaaprendizaje de la lengua inglesa es la que se establece entre los propios estudiantes. En este 


\section{AMAZÖNAS}

sentido, un lugar privilegiado lo ocupan las tareas que realizan entre sí de manera cooperada y el intercambio comunicativo que se logra establecer entre ellos durante la clase.

Dependerá en gran medida de la habilidad del profesor para ubicar a los estudiantes en situaciones comunicativas lo más cercanas a la realidad, en las que se establezcan relaciones interpersonales basadas en las buenas conductas, los principios y valores éticos que se corresponden con los patrones de la sociedad.

Se sugiere la orientación de tareas dirigidas al intercambio bilateral o trilateral de información, de ideas o valoraciones acerca del tema objeto de estudio en la clase. Esto permite al profesor comparar los puntos de vista de los estudiantes y detectar las insuficiencias que en el orden lingüístico y comunicativo pueden presentar y tomar, en consecuencia, las medidas adecuadas.

Al propiciar la interrelación estudiante-estudiante se les da la posibilidad de autoevaluarse y autocorregirse durante el propio acto comunicativo. Por otra parte, los que escuchan la intervención de sus compañeros tienen la oportunidad de evaluar la actuación de estos y participar en la corrección de los errores detectados, además contribuye a disminuir el miedo escénico y el temor a cometer errores durante el discurso.

\section{Carácter sistémico-comunicativo}

La pertinencia de la atención especial al sistema de los contenidos cognitivos de la enseñanza, así como la elaboración de tareas comunicativas para desarrollar la expresión oral en el idioma inglés se distingue por su carácter sistémico-comunicativo, el cual permite dirigir el proceso de enseñanza-aprendizaje del inglés con el fin de intercambiar significados en esta lengua en contextos funcionalmente organizados atendiendo a aspectos como la pronunciación, el vocabulario, la gramática, los estilos funcionales y diferentes registros de la lengua.

\section{Carácter educativo}

Concebir el proceso de enseñanza aprendizaje de la lengua inglesa posibilita no solo que los estudiantes adquieran un sistema de conocimientos y habilidades importantes para su formación integral como futuros profesionales, sino también de valores que trascienden en cualidades y actitudes personales positivas.

En ese orden, es permisible apuntar que los contenidos cognoscitivos establecidos a través de las tareas tienen un alto potencial educativo, porque están relacionados directamente con aspectos relevantes de la esfera cognitiva y socio-afectiva de la personalidad. Ello ofrece la posibilidad de que el profesor potencie el proceso valorativo de sus estudiantes con el objetivo de que estos continuamente y de manera consciente, aprecien el significado que los contenidos tienen desde el punto de vista social, ético, profesional o personal.

Aprender a comprender, aprender a planificarse en tiempo y aprender a convivir y cooperar con los demás, constituyen aprendizajes básicos que genera una amplia gama de valores entre los que sobresalen los referidos a el respeto así mismo y a los demás, la cooperación, la solidaridad, la independencia, la responsabilidad individual y colectiva.

\section{Carácter significativo vivencial del aprendizaje}

Se abordan todos y cada uno de los elementos, factores, condiciones que garantizan la adquisición, la asimilación y la retención del contenido por parte del estudiante, de modo que 


\section{AMAZđ̛́NAS}

adquiera significado propio, al considerar para ello como basamento teórico-metodológico las tareas comunicativas.

De acuerdo a este carácter, los nuevos conocimientos se incorporan en forma sustantiva en la estructura cognitiva del estudiante. Esto se logra cuando ellos relacionan los nuevos contenidos con los anteriormente adquiridos; pero es necesario que se interesen por aprender lo que se le está enseñando.

Con referencia a lo afectivo, es necesario conocer el nivel de motivación, necesidad e interés individual y colectivo que poseen los estudiantes para obtener mejores resultados en su aprendizaje, previendo así las limitaciones que surgen en el momento de adquisición del conocimiento, y al mismo tiempo tratar de buscar la solución de los mismos.

El sujeto es el constructor, el creador, el productor de su propio aprendizaje y no un mero reproductor del conocimiento de otros, lo que apoya la idea de que no hay aprendizaje amplio, profundo y duradero sin la participación activa del que aprende. Por lo tanto, no serían admisibles clases en que los estudiantes sean esencialmente receptores pasivos de la información proporcionada por el docente.

\section{Carácter con fines específicos}

El proceso de enseñanza-aprendizaje de lenguas extranjeras se concreta en una situación creada para que el estudiante aprenda a aprender. Se establece en un proceso dialéctico donde se crean situaciones para que el sujeto se apropie de las herramientas que le permitan operar con la realidad y enfrentar las tareas con una actitud creadora, personalizada y científica.

\section{Resultados y discusión}

Las tareas comunicativas que se proponen son significativas, no solo para motivar al estudiante, sino que constituye una parte fundamental en la construcción del conocimiento, la cual forma parte de los principales elementos para apoyar y enriquecer el aprendizaje, estas tienen la finalidad de llevar al estudiante a investigar, descubrir y construir, aproximándolo a la realidad y ofreciéndole ocasión para actuar.

Las tareas comunicativas en su proceso de elaboración se basaron en los temas de las 12 unidades de la serie face2face, nivel Pre-intermediate (B1), creada en el año 2012 por la editorial Cambridge University Press; libro que constituye la bibliografía básica del nivel B1. Los objetivos, temas, el léxico, la gramática y las funciones comunicativas están en consonancia con los descriptores correspondientes del MCERL.

En correspondencia con el estudio de las unidades, las autoras consideran que hay oportunidades para utilizar la expresión oral en todas las lecciones, puesto que existe la posibilidad de desarrollar el uso de funciones comunicativas con este objetivo.

Por tanto, para elaborar las tareas comunicativas se parte del carácter sistémico donde se presupone un conjunto de elementos estrechamente relacionados, sujetos a un ordenamiento lógico y jerárquico, el que asegura entre sus componentes la interdependencia que vincula a cada uno, con los restantes.

Los elementos básicos de la propuesta han sido estructurados en: tema, objetivo, nivel de asimilación, forma de comunicación y procedimientos, los cuales se corresponden con el carácter sistémico de las tareas comunicativas en el área de la enseñanza. 


\section{AMAZŹNAS}

Las autoras consideran necesario esclarecer las funciones de cada uno de estos aspectos, a través de su significado, en el contexto de esta investigación:

- El tema: es un asunto sobre el cual el estudiante se comunicará de forma oral, a través de actividades comunicativas adaptadas a la situación de comunicación del material lingüístico de la clase.

- El objetivo: constituye el punto de partida para el logro de los fines comunicativos o resultados previamente concebidos en el desarrollo de la tarea. Determina su contenido y el método que se utiliza. Son expresión de las situaciones comunicativas que se plantean en el nivel intermedio equivalente a un B1.

- El nivel de asimilación: se refiere al ordenamiento o articulación racional de las tareas, en una secuencia determinada por el grado de complejidad que requiere la comunicación por parte del estudiante, en el proceso de formación de hábitos y habilidades y la asimilación del material lingüístico.

- Forma de comunicación: define el grado de participación de cada estudiante en los planos individual y colectivo, durante la realización de la tarea.

- El procedimiento: facilita la aplicación del método y concreta las acciones y operaciones a realizar por los estudiantes en correspondencia con las exigencias de los objetivos y características de los contenidos de las tareas. Por otro lado, el procedimiento está directamente relacionado con las condiciones en que estas se desarrollan, además describe el contexto en que se realizan y los medios a utilizar en función del propósito.

Por lo consiguiente, para Aguilera (2018) se presentan algunos ejemplos de tareas comunicativas para el desarrollo de la expresión oral del idioma inglés, en los estudiantes de Ingeniería Geológica.

Task 1

Role playing

Topic: "Introductions and interviews"

Objective: practice the communicative functions of asking and answering questions about personal information, simulating a real situation.

Level of assimilation: production

Type of communication: pair work

Procedure: The whole class participates in this activity: a student assumes the role of the interviewed, while the rest of the students will function as interviewers. To succeed the task, students should look for more information about the subject matter and conceive the possible questions and answers. The task can be developed at the end of the topic "Introductions and interviews", since students have just acquired the necessary information and lexicon they need to talk about it, while working in a brief autobiographical paragraph telling who you are.

Situation:

Interview a student in your class. Take notes during the interview, and then introduce this student to the rest of the class or to a small group of classmates. Possible topics on interview follow. What questions might you ask to elicit these questions?

1. Name

2. Spelling name 


\section{AMAZđ̛́NAS}

3. Country of origin

4. Present residence

5. Length of time in (this city or country), both past and future

6. Reason for coming here

7. Field of study or work

8. Activities in free time

9. General well-being and adjustment to living here

10. Comments on living here

Activity A. Make an oral report telling who you are, what you have done in the past two years, and what your plans are for the next two years. Then exchange your paper with a classmate. Ask each other questions to clarify your understanding and elicit further information.

Activity B. Interview a classmate outside class. Make an oral report to your classmate.

Task 2

Topic: "Exams"

Objective: interact orally on the topic related to exams by asking and answering questions about different communicative functions.

Level of assimilation: production

Type of communication: pair work

Procedure: The task will be developed as part of the set of activities conceived to systematize lexicon-grammar fundamentals related to the best way to obtain good marks.

Students should arrange on pairs in order to do the activity. It consists on a dramatization of a conversation devised by them, following the rules this kind of task requires.

Situation:

- Your best friend hasn`t been doing very well in his/her exams lately. Give him/her advice.

Use these two pieces of advice:

- Have study groups, turn off the mobile while study.

- Think of two more pieces of advice and tell them to your friend

- You haven't been doing well in your exams lately, so you go to your best friend and ask him/her for some advice on what you can do to improve your results. not.

Respond to what he tells you, decide whether you are going to follow his/her advice or

Task 3. Minilecture

Topic: "Minerals"

Objective: to talk about the field of application of minerals showing a certain degree of independence.

Level of assimilation: production

Type of communication: group work 


\section{AMAZÓNAS}

Procedure: This task is assigned lessons before, so that students had plenty of time to arrange the information they need. The professor, together with the class, assigns the roles of the lecturer and participants.

Situation:

You were invited to offer a conference to beginners from Geological Engineering Major, about fields of application of minerals mining in La Veguita settlement. Offer your classmates part of it giving examples. Be aware that the audience will ask you some questions about the topic of your exposition.

Look for further information by consulting your professors. Presentation of the results of a research work

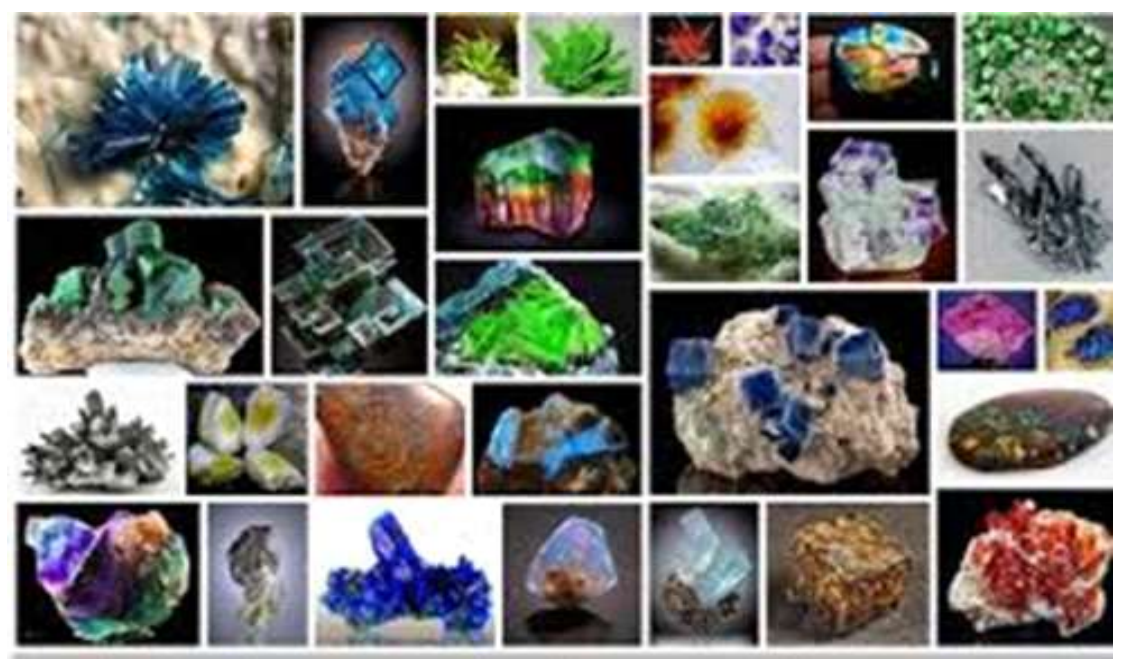

Task 4. Round Table about Types of rocks 1.

Topic: "Rocks"

Objective: to talk about the fields of application of types of rocks by using the necessary communicative functions demanded by the oral discussion of this topic.

Level of assimilation: production

Type of communication: group work

Procedure: The task is related to the reading "Rocks". Divide the class into groups of five/six students. This task can be combined with the activity 3 presented before.

Situation:

In the reading "Rocks", it is clearly shown, a wide field of application of the different types of rocks. Expand the information you have about this subject matter in a round table.

- Interview other persons if necessary.

- Consult other materials.

- Explain everything you know about igneous, metamorphic and sedimentary rocks.

Resulta oportuno manifestar que con la aplicación de las tareas comunicativas para desarrollar la expresión oral en el nivel Pre-intermediate B1 los estudiantes mejoran en gran 


\section{AMAZđ̛́NAS}

medida la competencia comunicativa, además de mostrar mayor motivación e interés por el aprendizaje del idioma inglés al acercarlos lo más posible a actos comunicativos reales.

Se puede expresar que las tareas comunicativas no están estáticas ni fijas en el tiempo sino por el contrario están inmersas en un proceso dinámico e interactivo en contante transformación y perfeccionamiento donde el método que utiliza el profesor con el estudiante es el resultado del proceso de interacción en su relación con el contexto, la labor educativa y la actitud hacia la enseñanza aprendizaje del idioma inglés.

\section{Conclusiones}

A partir de la interpretación del proceso de enseñanza-aprendizaje del desarrollo de la expresión oral del idioma inglés, al nivel equivalente a un B1, ha sido posible determinar las conclusiones siguientes:

Las consideraciones teóricas derivadas de la valoración de diferentes fundamentos epistemológicos, constituyen una vía para desarrollar la expresión oral del idioma inglés, al nivel intermedio equivalente a un B1.

La estructura de las tareas comunicativas que se proponen se concibe como una guía sujeta a cambios, por lo que permite su adecuación en diferentes situaciones comunicaciones vinculada a la realidad de la formación profesional de los estudiantes de la carrera Ingeniería Geológica y del Departamento de Idiomas.

Las tareas comunicativas propuestas constituyen un complemento del libro face2face nivel Pre-intermediate (B1) para desarrollar la expresión oral en inglés, las cuales han sido diseñadas para propiciar en los estudiantes los elementos de pronunciación, la fluidez y la exactitud, así como la utilización eficiente de funciones comunicativas características de este nivel.

\section{Referentes Bibliográficas}

Abbott, G. (1989). The teaching of English as an International Language: a Practical Guide. Ed. Revolucionaria, La Habana, p.57

Acosta, P. R. (1996). La enseñanza de lenguas extranjeras desde el contexto latinoamericano" Revista de Educación Mendive ISSN. 1815-7696 RNPS 2057. http://mendive.upr.edu.cu/index.php/MendiveUPR/article/view/1346

Aguilera, G. (2018). Tareas de aprendizaje para el perfeccionamiento de la expresión oral en inglés en la carrera de Ingeniería Geológica. Revista de Innovación Social y Desarrollo, 2 (2), 202-216.

Antich, R. (1986). The teaching of English in the Elementary and Intermediate level. La Habana: Ed. Pueblo y Educación.

Brown, H. D. (1994). Teaching by Principles: An Interactive Approach to Language Pedagogy. Englewood Cliffs, New Jersey.U.S.A.: San Francisco State University: Printice Hall Regents.

Byrne, D. (1989). Teaching Oral English. Ed. Revolucionaria, p.28.

Ellis, R. (2003). Task-based Language Learning. Oxford University Press.

Font, S. (2006). Metodología para la asignatura inglés en la secundaria básica desde una concepción polémica del enfoque comunicativo. (Tesis en opción al grado científico de Doctor en Ciencias Pedagógicas). Documento digitalizado, La Habana, p.20.

Howatt, A. (1984): A history of English language teaching. Oxford: Oxford University Press. 


\section{AMAZONANAS}

Medina, A. (2006). Didáctica de los Idiomas con Enfoque de Competencias: ¿Cómo enseñar el castellano y los idiomas extranjeros en cualquier nivel de educación? Barranquilla, Colombia: Ediciones CEPEDID.

Nunan, D. (1989). Designing Tasks for the Communicative Classroom. United Kingdom: Cambridge University Press, p. 12

Nunan, D. (2011). La enseñanza de lenguas basada en tareas. Nueva Edición. Editorial Edinumem. Cambridge de didáctica de lenguas.

Pujol-Breche, M. (1994). La Pedagogía del Texto: enseñanza-aprendizaje del producto final y del proceso de producción verbal. Revista de Psicología Social, Comunicación, Lenguaje y Educación. Madrid. España.

Richards, J.C. y Lockhart, C. (1995). Reflective Teaching in Second Language Classroom. Cambriage University Press.

Skehan, P. y Swain, M. (2001) "Researching Pedagogic Tasks", Second Language Learning, Teaching and Testing. $1^{\text {st }}$ Edition. Routledge. ISBN 9780582414822,

Willis, J. (2007). Doing Task- based Teaching. Oxford University. 